\title{
La lutte pour l'emploi et les alliances locales : le cas de Dominion Bridge à Lachine, Montréal
}

\author{
Jean-Marc Fontan, Juan-Luis Klein, Mylène Tremblay \\ CRISES - Université du Québec à Montréal
}

Le 10 septembre 1998, la Dominion Bridge de Lachine déclare faillite. Près de 300 travailleurs perdent leur emploi. Un mois après la fermeture, suite à l'effort conjugué du syndicat et de la communauté locale, Dominion Bridge est rachetée par Au Dragon Forgé, une entreprise de Terrebonne spécialisée dans les structures d'acier et les métaux ouvrés.

Il est fréquemment affirmé que les activités économiques dépendent uniquement des lois du marché. L'analyse de l'évolution récente d'une entreprise montréalaise - Dominion Bridge - nous montre que ces lois peuvent être influencées par la collectivité. L'étude de la mobilisation pour sauver les emplois de la Dominion Bridge nous permet de mettre en valeur l'apport de l'action sociale dans le maintien des activités productives d'une entreprise dans le but de sauver des emplois. Cette étude s'insère dans un programme de recherche plus large qui concerne les changements dans l'action collective syndicale, et notamment les actions que les syndicats mènent dans le but d'assurer la viabilité du développement économique au niveau local ${ }^{1}$.

En attirant l'attention sur l'apport des actions collectives syndicales, nous ne prétendons pas démontrer que les lois du marché peuvent être toujours et constamment détournées par des stratégies sociales volontaristes. Notre analyse n'a pas cet objectif. Toutefois, elle met en lumière la capacité des organisations sociales, voire des mouvements sociaux, d'influencer les acteurs publics et privés dans l'évaluation des facteurs qui président à leur prise de décision en ce qui concerne la localisation des activités productives.

\section{Récit d'une mort annoncée}

En 1994, la section locale 2843 du Syndicat des Métallos, membre de la Fédération des travailleurs et travailleuses du Québec (FTQ), s'inquiète du fait que les nouveaux propriétaires de la Dominion Bridge sont plus soucieux de réaliser des profits à court terme que d'honorer de façon satisfaisante les contrats de la société. Les Métallos font alors appel au service Urgence-Emploi du conseil régional de la FTQ ${ }^{2}$.

Le service Urgence-Emploi a vu le jour le 2 mars 1992. Agissant à titre de conseiller auprès de syndicats affiliés à la FTQ, Urgence-Emploi aide ces derniers à prévenir les licenciements collectifs et les fermetures d'entreprises (une démarche préventive pour le maintien et la sauvegarde de l'emploi) ou les appuie, dans le cas de licenciements ou de fermetures, pour mettre sur pied des comités de reclassement (démarche de réintégration à l'emploi). Dans cette mesure, ce service illustre les changements du mouvement syndical en ce qui concerne les rapports avec l'employeur et montre l'importance que ce mouvement accorde à la conservation des emplois. Il s'agit d'un outil qui apporte une aide cruciale aux syndicats locaux qui veulent analyser l'état de santé de leur entreprise.

Selon la méthode développée par Urgence-Emploi, tout diagnostic d'une entreprise indiquant des signes de dévitalisation servirait de base à la réalisation d'un plan d'action pour assurer une relance des activités 
de l'entreprise. Dans le cas de Dominion Bridge, le plan d'action qui fut établi permettait au syndicat local d'identifier des pistes d'intervention et des actions à appliquer, de concert avec les dirigeants de l'entreprise, pour tenter d'éviter la fermeture de cette dernière.

De 1994 à 1998, malgré le diagnostic réalisé par le syndicat local et les discussions avec les dirigeants de la Dominion Bridge, la situation de l'entreprise se dégrade lentement. Les propriétaires font fi des propositions syndicales. Ils adoptent une stratégie défensive reposant sur un optimisme de façade lié à l'impact imminent que devait avoir la signature de contrats alors en négociation. Mais, malgré 1'expectative de ces contrats, les actionnaires et le conseil d'administration de la Dominion Bridge sont divisés sur l'orientation et la gestion immédiate de l'entreprise. Le syndicat est mis au courant de la situation tendue qui prévaut à la direction. En avril 1998, à cause de l'accumulation des licenciements, le syndicat convoque les médias. Il rend publique la situation prévalant dans l'usine. Aux yeux du syndicat local, la crise de leadership mettait en péril l'avenir de la compagnie.

L'évolution de la crise donne raison au syndicat, dans les mois qui suivent, et la Dominion Bridge est confrontée à d'insurmontables problèmes de liquidité. Le 11 août 1998, l'inévitable se réalise : la Dominion Bridge se place sous la protection de la Loi sur les faillites.

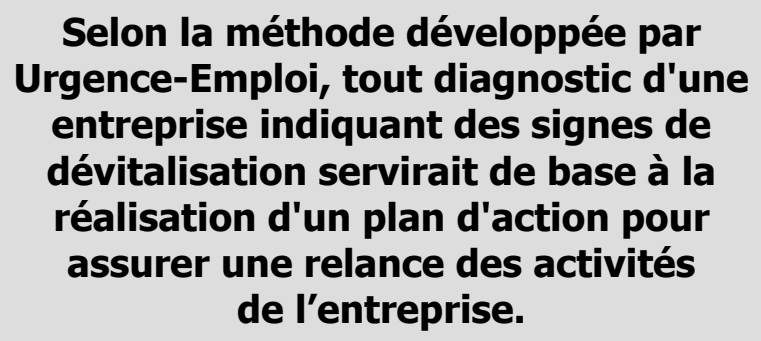

\section{Alliance avec la communauté et création d'un Comité de relance}

En août 1998, pressentant la fermeture de l'entreprise, le syndicat local s'associe à une corporation de développement économique communautaire, à savoir Transaction pour l'emploi. Cette corporation regroupe les forces économiques et communautaires des anciennes villes Lasalle, Lachine et Saint-Pierre. L'analyse de la situation conduit la directrice de Transaction pour l'emploi et les leaders du Syndicat local des Métallos de la Dominion Bridge à s'entendre sur la formation d'un Comité de relance qui regrouperait une diversité d'acteurs impliqués dans le développement de la communauté.

\section{Organisations membres du Comité de relance de la Dominion Bridge}

- Syndicat des Métallos (section locale 2843 de la FTQ)

- Conseil du travail du Montréal métropolitain

- Transaction pour l'emploi

- Société de développement des artères commerciales de Lachine

- Chambre de commerce du sud-ouest de l'île de Montréal

- Regroupement des gens d'affaires de Lachine

- Commission scolaire Marguerite-Bourgeois

- Cégep André-Laurendeau

- Ville de Lachine

- Députés fédéral et provincial

- Organisation locale du PQ 
Les représentants du Comité de relance, sous le leadership du syndicat local, esquissent les premiers éléments d'une opération de relance des activités de la Dominion Bridge. Dans un premier temps, la stratégie élaborée consiste à mobiliser les ressources syndicales. Ces dernières sont composées des travailleurs de l'entreprise. Ils représentent la clé du succès pour sensibiliser, à partir de manifestations publiques, la population et les gouvernements.

Dans un deuxième temps, le Comité rallie à sa cause des intervenants locaux des milieux politique, communautaire et privé. Le journal local est ciblé. Les militants se donnent pour mission d'informer la population locale par la distribution de feuilles volantes expliquant les tenants et aboutissants de la bataille opposant les travailleurs aux dirigeants de l'entreprise.

L'alliance entre le syndicat local et les différents secteurs de la communauté est facilitée par le travail de Transaction pour l'emploi et par le caractère hautement symbolique de la Dominion Bridge. Rappelons que l'ancienne ville de Lachine s'est construite autour de cette entreprise. La majorité des résidents ont eu au moins un membre de leur famille, un voisin ou un ami qui y ont travaillé.

Le fort sentiment d'appartenance de la ville à l'entreprise explique certainement pourquoi le Comité de relance a réussi à mobiliser, sur une large base, une diversité de représentants. D'autant plus que cette région de l'île de Montréal connaissait depuis une dizaine d'années une vague importante de fermetures d'usines ${ }^{3}$. La collectivité se trouvait donc, une fois, de plus affectée par de nouvelles pertes d'emplois.

\section{Pour se faire entendre et comprendre : l'action politique}

Dans cette lutte pour l'emploi, une décision clé prise par le syndicat local a été d'agir sur les preneurs de décisions, notamment les gens d'affaires. Le syndicat local avait peu d'outils pour les mobiliser. Par contre, par l'intermédiaire d'autres grands preneurs de décisions, des ministres et des chefs syndicaux, ils pouvaient espérer faire bouger le milieu des affaires.
La directrice de Transaction pour l'emploi a contacté rapidement les principaux élus locaux des trois paliers de gouvernement. Ces derniers ont accepté de joindre les rangs du Comité de relance. Tant le maire de Lachine que les députés provincial et fédéral ont joué un rôle important au sein du comité. La directrice de Transaction pour l'emploi a réussi à harmoniser les actions prises par le syndicat local aux ressources regroupées au sein du comité local.

Le syndicat local a quant à lui exercé des pressions sur l'appareil syndical. Dans un premier temps, un travail de sensibilisation a été fait auprès de l'agent de développement du Fonds de solidarité chargé du dossier de la Dominion Bridge. Une première analyse interne indiquait que la relance de l'entreprise serait vouée à l'échec. Les représentants du syndicat local ont alors décidé de faire des pressions auprès de la haute direction et du Fonds de solidarité et de la centrale FTQ.

La jonction entre les deux pôles d'action - communautaire et syndical - a permis d'interpeller directement les gouvernements provincial, via Lucien Bouchard et Bernard Landry, et fédéral, par l'intermédiaire de Jean Chrétien, Martin Cauchon et Paul Martin. Les membres du comité ont pu rencontrer le ministre fédéral des Finances, Paul Martin, à 24 heures d'avis, et, à deux reprises, le Vice-premier ministre de l'époque, Bernard Landry.

\section{Les militants se donnent pour mission d'informer la population locale par la distribution de feuilles volantes expliquant les tenants et aboutissants de la bataille opposant les travailleurs aux dirigeants de l'entreprise.}

Le 2 septembre 1998, le Comité de relance organise une première manifestation. Celle-ci est un succès et se traduit par une excellente couverture médiatique. Cette action n'empêche toutefois pas l'inévitable : le 10 septembre, tel qu'indiqué précédemment, la compagnie déclare faillite. Le syndicat local l'apprend par la voie des journaux. L'atmosphère est alors extrêmement tendue. Les travailleurs licenciés effectuent une opération essoufflante de piquetage devant l'usine, sur une durée de douze semaines, afin 
d'empêcher la saisie des équipements, de l'inventaire et des pièces ouvrées des contrats en cours. Ils s'y relaient sans relâche, sept jours sur sept. Dès 5 heures du matin, plusieurs d'entre eux sont sur place. "Mieux vaut se pointer à l'usine comme à l'ordinaire que de rester chez soi à broyer du noir ", jugent-ils (entrevues). Pour les travailleurs de la Dominion Bridge, il ne s'agit pas d'une simple grève pour réclamer de meilleures conditions de travail ou de meilleurs salaires. C'est d'une fermeture dont il est question, entraînant une perte durable d'emplois.

\section{Tant le maire de Lachine que les députés provincial et fédéral ont joué un rôle important au sein du comité.}

La semaine suivante, une autre manifestation est organisée. Elle regroupe des sympathisants et des ouvriers de la Dominion Bridge. Ces derniers bloquent l'accès d'un petit pont reliant Lachine à Ville Lasalle. Les députés provincial et fédéral qui siègent au Comité de relance avaient parallèlement convoqué les médias pour une conférence de presse. Les dirigeants syndicaux profitent de cette conférence pour diriger la manifestation là où se déroulait le point de presse. La visibilité souhaitée auprès des médias électroniques est atteinte.

La plus importante manifestation a lieu le 17 septembre. Les travailleurs et sympathisants sont convoqués par le syndicat local et le Comité de relance devant l'usine. Deux autobus de la Commission de transport de Montréal attendent les manifestants : destination, le pont Mercier. Ce dernier a une double valeur symbolique. Il a été construit à partir d'infrastructures de métal produites à la Dominion Bridge en 1948-1949. Le pont rappelle aussi les événements entourant la crise autochtone du début des années 1990.

Vers 10 heures du matin, une centaine de manifestants débarquent sur le pont Mercier. Pour attribuer à ce geste un sens historique, nombre de manifestants se parent de foulards. Ils bloquent une voie de l'autoroute, en direction sud durant 45 minutes dans le but d'attirer l'attention des médias pour exercer des pressions sur les gouvernements. Pendant cette occupation du pont, les manifestants distribuent des feuilles volantes expliquant la cause du conflit. La police provinciale intervient. Les manifestants repartent donc en autobus, en direction cette fois du bureau du ministre fédéral des Finances et député de Lasalle-Émard, Paul Martin.

\section{Implication de ministres et du Fonds de Solidarité}

Le cumul d'actions menées par le mouvement de mobilisation pour sauver les emplois de la Dominion Bridge commence à porter fruit. Le blocage du pont Mercier fait mouche dans l'opinion publique. Plusieurs élus se font alors entendre. Les ministres Paul Martin et Bernard Landry se montrent déterminés à appuyer et à faciliter tout projet d'acquisition de la Dominion Bridge par une autre entreprise. Parallèlement, le Fonds de solidarité de la FTQ, fortement sollicité par la direction des Métallos, et ce dès le début de la crise, décide de s'impliquer dans la relance de l'usine.

L'implication du Fonds de solidarité dans le dossier constitue un point tournant pour le déblocage de la crise. Il est vrai que cette intervention s'inscrit tout naturellement dans la mission première de l'organisme, mais celui-ci avait refusé au départ de s'impliquer dans le sauvetage de l'usine. Cependant, après plusieurs démarches et négociations avec les entrepreneurs qui se montrent intéressés par le rachat de la Dominion Bridge, une proposition viable de rachat venant d'une PME crée des espoirs. Le Fonds de solidarité s'associe alors avec la firme Au Dragon Forgé, une entreprise familiale de Terrebonne, pour remettre en opération l'usine de Lachine. Les dirigeants d'Au Dragon Forgé sont disposés à investir 6 à 10 millions de dollars pour redémarrer les opérations. Le Fonds en injectera près de 8 millions. Un seul problème reste à résoudre : le dossier de contamination des terrains de la Dominion Bridge, laquelle contamination est considérée comme lourde. Le gouvernement finit par accorder une aide financière afin de réaliser les travaux de décontamination. L'heureux dénouement est annoncé en conférence de presse par le Comité de relance le 12 novembre 1998, soit trois mois après le début de la mobilisation syndicale. 


\section{Action collective et développement local}

Le développement local est défini comme l'ensemble des initiatives mises de l'avant par des acteurs locaux pour dynamiser le marché local. Ces initiatives locales reposent fondamentalement sur une mobilisation de ressources internes et externes à la communauté dans le but de créer des emplois afin de générer de la richesse. Les défenseurs les plus progressistes du développement local se donnent aussi pour objectif de travailler à une redistribution équitable de l'emploi et de la richesse au niveau local afin d'assurer une amélioration de la qualité de vie de l'ensemble de la population.

Parmi l'ensemble des initiatives de développement, celles visant le développement entrepreneurial privilégient habituellement une stratégie de création de nouveaux emplois. L'analyse du cas de la Dominion Bridge montre qu'il est non seulement possible mais très souhaitable d'adopter une stratégie complémentaire de protection et de croissance du bassin d'emplois d'un territoire local. Comment? En créant des outils, des mesures ou des mécanismes propices au maintien en activité et à la croissance de l'emploi au sein de moyennes et de grandes entreprises locales ${ }^{4}$.

Un point de vue bien partagé par les courants dominants de la science économique affirme que les entreprises désuètes n'ont pas à être aidées et qu'elles sont vouées à disparaître. Selon cette logique de pensée, les lois du marché, plus particulièrement de la concurrence, jouent en faveur d'un renouvellement naturel du tissu économique. Les bonnes activités remplaceraient naturellement les mauvaises, selon un principe similaire à celui en œuvre dans la sélection naturelle des animaux ou des plantes. Les cycles économiques feraient en sorte que dix emplois perdus dans l'économie en déclin seraient remplacés par dix emplois ou plus dans l'économie en émergence. Selon cette façon de penser, toute intervention proactive en matière de développement de l'emploi devrait principalement dédier les ressources publiques ou privées aux projets visant le renouvellement des bassins d'entreprises. Il s'agit uniquement d'appuyer l'émergence et le développement de nouvelles entreprises au détriment d'actions d'assistance d'entre- prises obsolètes, lesquelles finiraient de toute façon par disparaître.

De 1900 à 1970, l'évolution cyclique de l'économie des pays développés concordait avec ce raisonnement. En gros, les crises, comme celle des années 1930, permettaient de nettoyer le paysage entrepreneurial. La crise des années 1930 s'est traduite par un renouvellement des secteurs moteurs, lequel a favorisé une longue période de croissance connue sous le nom des « Trente glorieuses ».

\section{Le développement local est défini comme l'ensemble des initiatives mises de l'avant par des acteurs locaux pour dynamiser le marché local.}

Dès le début des années 1980, une nouvelle dynamique est en place. Il devient beaucoup plus difficile de créer des emplois et il faut de plus en plus de ressources pour générer des emplois bien rémunérés et présentant de bonnes conditions de travail. Dans ce nouveau contexte, l'emploi devient un bien rare. Cette situation pousse à un double effort d'innovation: un premier, centré sur l'innovation économique, issu d'entrepreneurs appelés à renouveler les pratiques entrepreneuriales; un deuxième, issu de promoteurs du développement local, faisant appel à l'innovation sociale pour faciliter le maintien en activité d'entreprises obsolètes. Tant les premiers que les deuxièmes bousculent les façons de faire des derniers en invitant à revoir les façons d'entreprendre ou de développer le territoire.

La lutte pour le maintien des emplois à la Dominion Bridge s'inscrit dans un contexte de redéfinition des pratiques d'intervention en matière de développement économique local. Elle constitue un exemple du refus manifesté par des intervenants du sud-ouest de Montréal de voir le bassin d'emploi de leur territoire se transformer progressivement en bassin de personnes sans emploi.

L'étude des mobilisations découlant d'une douzaine de situations de fermetures d'usines et l'analyse en profondeur du cas de relance de la Dominion Bridge nous permettent de dégager quelques constats sur les 
mesures à prendre pour protéger les bassins d'emplois ${ }^{5}$.

Un premier constat a trait à la dynamique économique qui s'implante à partir des années 1980. Les études que nous avons menées indiquent qu'aucune grande entreprise, de l'ancienne ou de la nouvelle économie, n'est protégée contre un licenciement collectif d'importance ou n'est à l'abri d'une fermeture.

Nous avons rencontré des cas de fermeture d'une filiale d'une multinationale alors que sa technologie était à la fine pointe de l'innovation, que ses travailleurs étaient très qualifiés, que la productivité d'ensemble était élevée et que le marché pour les produits réalisés était en demande : le cas de l'usine Philipps à Saint-Jérôme. Malgré tous ces facteurs, l'usine ferme. Pourquoi ?

\section{L'étude des mobilisations découlant d'une douzaine de situations de fermetures d'usines et l'analyse en profondeur du cas de relance de la Dominion Bridge nous permettent de dégager quelques constats sur les mesures à prendre pour protéger les bassins d'emplois.}

Dans le cas de Philipps, les raisons invoquées par les dirigeants de l'entreprise fermée sont simples. L'usine cesse ses opérations car les capacités de production de la multinationale sont telles qu'il lui devient impératif de fermer une ou plusieurs de ses unités mondiales. Il le faut, non pas parce que les usines ne sont pas rentables, mais parce que le taux global de profit n'est pas assez élevé pour les actionnaires.

Les turbulences que nous observons présentement dans les secteurs de pointe de l'économie québécoise (aéronautique, pharmaceutique, informatique, télécommunications, etc.) vont d'ailleurs dans ce sens. En optant pour une rationalisation, les impératifs financiers de rendement forcent une réduction du plancher de production à partir d'une concentration des entreprises et d'une réduction du nombre de filiales.
Dans le cas de la Dominion Bridge, la situation de fermeture tenait moins à un problème de surcapacité de production qu'à un problème de mauvaise gestion d'une usine acquise par des entrepreneurs ayant des compétences, mais pas celles nécessaires pour relever le défi qu'ils s'étaient donnés. Lorsque le désir de profit à très court terme l'emporte sur le désir de stabilisation d'un taux de profit moyen à long terme, la grande entreprise, comme unité de production, devient un actif géré uniquement en fonction de sa valeur financière de revente. Pour les principaux actionnaires de la Dominion Bridge, la liquidation des avoirs est rentable. Pour les travailleurs, la population locale et le réseau économique régional, une telle pratique signifie des pertes économiques et surtout des coûts sociaux importants.

Évidemment, les cas de fermetures d'entreprises désuètes dont les facteurs de production sont d'une autre époque et le bassin d'emplois déqualifié, ou les cas de fermetures d'entreprises dont les facteurs de production sont en voie d'obsolescence sont plus facilement prévisibles. Les outils développés par certaines centrales syndicales, dont la FTQ, permettent aux syndicats locaux d'établir relativement facilement, sans recourir à des ressources importantes, des diagnostics sur l'état de santé de leur entreprise. De là il devient envisageable, si la situation est comprise à temps et que les conditions le permettent, de mettre en place des mécanismes de redressement.

En résumé, les fermetures peuvent affecter tout aussi bien des entreprises en santé que des entreprises malades ou en voie de l'être. Ces fermetures peuvent être contrées lorsque le syndicat local voit venir le coup ou lorsqu'il se mobilise dès l'annonce de la fermeture autour d'un plan de relance.

Bien sûr, toutes les mobilisations ne mettent pas en scène les mêmes mécanismes d'action pour assurer la survie de l'entreprise. Nos travaux indiquent qu'il n'y a pas de recette unique ni de victoire assurée. Ils font état aussi d'une différence importante entre des situations de fermeture d'entreprises publiques et d'entreprises privées. Nous n'élaborerons toutefois pas sur ce point. 
Les mobilisations étudiées révèlent la présence de deux processus complémentaires d'action. Un premier tient à la mobilisation du milieu syndical par le syndicat local. Il importe que ce dernier assume un leadership fort et qu'il établisse rapidement un diagnostic clair et un plan d'action proactif et non pas passif. Le pire scénario que nous avons rencontré est celui de représentants de syndicats locaux qui espèrent que les choses vont s'arranger d'elles-mêmes, que la situation va revenir à la normale par les actions menées par le dirigeant de l'entreprise.

\section{En résumé, les fermetures peuvent affecter tout aussi bien des entreprises en santé que des entreprises malades ou en voie de l'être.}

Dans certains cas, le dirigeant de l'entreprise menacée de fermeture est en mesure de redresser la situation. Dans nombre d'autres cas, il tente le maximum, mais il est rapidement dépassé par la situation. À ce moment, il est souvent trop tard pour d'autres interventions. La fermeture devient alors inévitable et les chances de rachat, pas toujours évidentes.

Le cas de la Dominion Bridge indique que, même lorsque la faillite est déclarée, il est possible de trouver une voie positive de sortie de crise. Toutefois, toutes les entreprises ne présentent pas le même profil, en termes d'actifs ou de potentiel, que celui retrouvé chez l'ex Dominion Bridge.

Une fois le leadership du syndicat local bien ancré et la mobilisation des ressources syndicales suffisamment amorcée par le syndicat local, nous avons observé, dans nombre de cas, le besoin d'élargir la mobilisation à d'autres acteurs locaux, dont des ressources extérieures à la communauté affectée par la fermeture. Nos études montrent que la mobilisation de ressources syndicales par le syndicat local n'est pas gagnée d'avance et que souvent, le succès de cette dernière dépend de la capacité des acteurs syndicaux de gagner l'appui de ressources externes. Ainsi, la participation du Fonds de solidarité dans une activité de relance d'entreprise n'est pas automatique. La mission du Fonds répond certes à des objectifs syndicaux, mais elle tient aussi compte d'impératifs économiques. Le rendement des actifs du Fonds doit être positif.

Le syndicat local peut aider sa cause et faciliter la participation de fonds syndicaux en assurant à ces derniers l'implication financière des paliers gouvernementaux dans la relance de l'entreprise en situation de fermeture. L'exemple de la Dominion Bridge est éclairant, comme le fut aussi celui de l'usine Kenworth à Sainte-Thèsèse. Le fait que les gouvernements aient été sensibilisés puis impliqués dans le projet de relance facilite grandement la participation de fonds privés ou de fonds syndicaux. L'injonction de fonds publics dans la relance de ces deux entreprises est une condition importante pour que se mette en place un montage financier gagnant.

Ce dernier point ouvre la voie au constat suivant : la mobilisation de ressources syndicales ne suffit pas pour relancer les activités d'une entreprise en très grande difficulté. Cette mobilisation a tout à gagner en élargissant ses bases pour inclure des ressources communautaires, institutionnelles, publiques et privées. Dès lors, la grande leçon de notre étude tient au fait que, sur le long terme, et en fonction de la nouvelle compétitivité économique, les syndicats locaux ont intérêt à développer des alliances avec d'autres acteurs locaux, dont les élus des trois paliers de gouvernement. Cette mobilisation peut impliquer le réseau des organismes communautaires, le réseau institutionnel, les commerçants et les entrepreneurs locaux. Elle peut aussi bénéficier du support de la population et des médias locaux ou nationaux.

\section{Le cas de la Dominion Bridge indique que, même lorsque la faillite est déclarée, il est possible de trouver une voie positive de sortie de crise.}

\section{Conclusion}

Quels apprentissages dégager des actions collectives vouées à la sauvegarde d'emplois ? Un premier apprentissage tient certes au continuel travail de vigilance et à la nécessité d'innover au plan des modalités d'intervention lorsque des acteurs entendent réagir de façon proactive aux turbulences créées par 
le marché. Vigilance et innovation ne vont pas de soi; sans leadership fort, point d'espérance de victoire, comme l'enseigne l'étude du cas de la Dominion Bridge. Dès lors, les communautés disposant d'un bassin d'emplois manufacturiers important ont tout à gagner à établir des passerelles de communication entre leurs milieux d'intervention de façon qu'au moment opportun, des fronts communs et des alliances puissent rapidement mettre à la disposition de la communauté les outils nécessaires pour faire face à une crise de l'emploi.

Un deuxième apprentissage, révélé par les actions menées par les Métallos de la Dominion Bridge, tient au fait que les problèmes et les solutions en matière de création et de maintien des emplois ont certes une dimension technique, mais avant tout ils ont une dimension sociale et humaine. Dès lors, il est possible d'intervenir sur les problèmes, car les solutions ne sont pas inexorablement hors de portée.

Enfin, pour les acteurs du développement local, il apparaît clairement que la mobilisation de ressources représente un curriculum d'aptitudes et de compétences incontournables. Acquérir du savoir-faire en la matière est une opération continue qui se construit au jour le jour. Ce travail de construction dépasse largement le cadre d'une lutte pour la relance d'une entreprise. Il permet aussi à une communauté de se doter d'outils de prévention et de mécanismes efficaces de réaction. Ce faisant, globalement, la capacité d'action d'une communauté sur elle-même augmente. Cette capacité d'action devient ou forme un capital socio-territorial, lequel constitue une configuration propice au développement local.

\section{Les problèmes et les solutions en matière de création et de maintien des emplois ont certes une dimension technique, mais avant tout ils ont une dimension sociale et humaine.}

\section{Notes et références}

1 Pour les principaux résultats de ce programme de recherche, voir: Fontan, J.-M. et J.-L. Klein (1999). Pour un meilleur rapport de force, les alliances locales (les luttes syndicales ne se gagnent plus seules), Montréal, Fonds de solidarité FTQ, Fédération des travailleurs et travailleuses du Québec et Service aux collectivités de l'UQÀM, 30 p.; Fontan, J.-M. et J.-L. Klein (2000). « Mouvement syndical et mobilisation pour l'emploi : renouvellement des enjeux et des modalités d'action », Politique et société, vol. 19, $n^{\text {o }}$ 1, p. 79-102; Klein, J.-L. et J.-M. Fontan (2002). Syndicats et communautés dans la gouvernance locale : une recherche exploratoire sur Montréal, Recherches sociographiques (sous presse).

2 Les éléments présentés dans cette section reprennent les grandes lignes de la monographie réalisée par Mylène Tremblay sur la relance de la Dominion Bridge (Tremblay, 2000). Un vidéo produit pour le Fonds de solidarité et la FTQ par André Vanasse décrit la lutte du syndicat local et de Transaction pour l'emploi afin de relancer l'usine (Vanasse, A. 2001. Unis pour sauver des emplois : la lutte à la Dominion Bridge (1994-2000), Les productions Bonsaï, Fonds de solidarité FTQ et Fédération des travailleurs et travailleuses du Québec, vidéo de 42 minutes.). Les informations sur la CDÉC de LaSalle-Lachine sont disponibles sur le site :

http://transaction-emploi.qc.ca:9880/mission.htm.

Sur le service urgence emploi, voir l'information présentée dans le portail de la FTQ :

www.ftq.qc.ca/ftq_en_region/montreal/suite.asp?aid=1822.

3 Mentionnons, entre autres, le cas des fermetures des entreprises Jenkins Valve, Valmet, GE et Sulzer.

4 Une telle stratégie, adoptée par exemple par une Corporation de développement économique communautaire - le Regroupement pour la relance de l'économie et de l'emploi du sud-ouest de Montréal -, permet de cibler des interventions auprès des entreprises manufacturières de 50 employés et plus de son territoire (voir la page Web du RESO : www.resomtl.com).

5 La première étude a été effectuée de 1998 à 1999. Elle a bénéficié d'un financement de la Fondation d'éducation et de formation économique du Fonds de solidarité de la FTQ. La deuxième est présentement en cours. Elle bénéficie d'un financement du Centre national de recherche en sciences humaines du Canada. 